\title{
Improvement of Learning and Memory Induced by Cordyceps Polypeptide Treatment and the Underlying Mechanism
}

\author{
Guangxin Yuan $\left(\mathbb{D}\right.$, Liping An $\left(\mathbb{D}\right.$, Yunpeng Sun $\mathbb{D}^{D}$, Guangyu Xu $\mathbb{C D}^{\circ}$, and Peige Du $(\mathbb{D}$ \\ College of Pharmacy, Beihua University, Jilin 132013, China \\ Correspondence should be addressed to Guangyu Xu; xuguangyu2005@163.com and Peige Du; dupeige2001@126.com
}

Received 20 September 2017; Revised 25 January 2018; Accepted 12 February 2018; Published 15 March 2018

Academic Editor: Kuzhuvelil B. Harikumar

Copyright ( 2018 Guangxin Yuan et al. This is an open access article distributed under the Creative Commons Attribution License, which permits unrestricted use, distribution, and reproduction in any medium, provided the original work is properly cited.

\begin{abstract}
Our previous research revealed that Cordyceps militaris can improve the learning and memory, and although the main active ingredient should be its polypeptide complexes, the underlying mechanism of its activity remains poorly understood. In this study, we explored the mechanisms by which Cordyceps militaris improves learning and memory in a mouse model. Mice were given scopolamine hydrobromide intraperitoneally to establish a mouse model of learning and memory impairment. The effects of Cordyceps polypeptide in this model were tested using the Morris water maze test; serum superoxide dismutase activity; serum malondialdehyde levels; activities of acetyl cholinesterase, $\mathrm{Na}+\mathrm{k}+$-ATPase, and nitric oxide synthase; and gamma aminobutyric acid and glutamate contents in brain tissue. Moreover, differentially expressed genes and the related cellular signaling pathways were screened using an mRNA expression profile chip. The results showed that the genes Pik3r5, Il-1 $\beta$, and Slc18a2 were involved in the effects of Cordyceps polypeptide on the nervous system of these mice. Our findings suggest that Cordyceps polypeptide may improve learning and memory in the scopolamine-induced mouse model of learning and memory impairment by scavenging oxygen free radicals, preventing oxidative damage, and protecting the nervous system.
\end{abstract}

\section{Introduction}

Learning and memory are among the main functions of the human brain, and together they play an important role in biological evolution and development [1]. Learning and memory are higher physiological activities in the brain and also the core components of cognitive function [2]. With increasing age, declining learning and memory abilities represent a common phenomenon and most of the population has been affected by hypomnesia [3]. Moreover, cognitive deficits develop during the progression of various neurological diseases, including Alzheimer's disease (AD) and Parkinson syndrome (PD) [4]. Therefore the early prevention and treatment of these symptoms of declining learning and memory have attracted much attention, in part due to their very broad potential market [5].

At present, the drugs commonly used clinically for prevention and improvement of learning and memory impairment include free radical scavengers, drugs to prevent beta amyloidal deposits formation, $\mathrm{M}$ receptor agonists, and acetyl cholinesterase inhibitors. Unfortunately, these few types of drugs used to treat learning and memory impairment commonly present poor effects regarding learning memory, toxicity, adverse side effects, and no preventive effect against the occurrence and progression of neurological diseases. Traditional Chinese medicines have shown some advantages over the traditional drugs for diseases involving learning and memory impairment [6-8], such as fewer and less severe side effects, more action targets, and low cost. Thus, there is much interest in identifying effective drugs among traditional Chinese medicines for the prevention and improvement of impaired learning and memory. Cordyceps militaris (North Cordyceps sinensis) is a rarely used Chinese herbal medicine at present, with bioactivities such as improvement of immunity [9-11], bacteriostasis [12, 13], and antihypertension [14]. Previous studies by our research group demonstrated that Cordyceps militaris can improve the learning and memory, and its main active ingredient is likely its polypeptide complexes [15-17].

To investigate the mechanism responsible for this effect of Cordyceps militaris on learning and memory, in the present study, we first established a mouse model of acquired learning 
and memory impairment via intraperitoneal injection of scopolamine hydrobromide. Using this model, we examined the effects of Cordyceps polypeptide on the learning and memory abilities of the mice by observing mouse behavior serum superoxide dismutase (SOD) activity; serum malondialdehyde (MDA) content; the acetyl cholinesterase AChE, $\mathrm{Na}^{+}$$\mathrm{k}^{+}$-ATPase, and endothelial nitric oxide synthase (eNOS) activities; and the gamma aminobutyric acid (GABA) and glutamate (Glu) contents in mouse brain tissues. Furthermore, mice were treated with Cordyceps polypeptides prepared via an enzymolysis approach, and an mRNA expression microarray was used to screen the differentially expressed genes and related signaling pathways in the brain tissues of treated mice. Overall, the ability of Cordyceps polypeptide to prevent and improve learning and memory impairment in the model mice was confirmed, and the data obtained for the related mechanisms may provide an important theoretical basis for the further research and development of Cordyceps polypeptide.

\section{Experimental Materials and Methods}

\subsection{Experimental Materials}

2.1.1. Animals. A total of 120 eight-week-old male ICR mice (specific-pathogen free grade), weighing 18 22 g, were purchased from Liaoning Changsheng Biotechnology Co., Ltd. (license number: SCXK (Liao)-2015-0001). The mice were housed individually in an environment of controlled temperature $\left(20 \pm 1^{\circ} \mathrm{C}\right)$ and humidity $(40-70 \%)$ and subjected to $12 \mathrm{~h}$ light/dark cycle with free access to food and water. The study was conducted according to the European Community Guidelines for the Use of Experimental Animals, and the Ethics Committee of Beihua University approved the study protocol.

2.1.2. Cordyceps militaris. Fruiting bodies of Cordyceps militaris were purchased from Shenyang Nizi Cordyceps Cultivation Base.

2.1.3. Reagents and Instruments. Piracetam Tablets (Northeast Pharmaceutical Group Shenyang First Pharmaceutical Co. Ltd; batch number: 5141224); scopolamine hydrobromide (Chengdu Manchester Stewart Biological Technology Co. Ltd., batch number: 150417) and pepsin (activity: 3000 $3500 \mathrm{u} / \mathrm{mg}$; Beijing Dingguo Changsheng Biotechnology Co., Ltd.); acetylcholinesterase (AChE), $\gamma$-aminobutyric acid (GABA), Na+-K+-ATPase, glutamic acid (Glu), monoamine oxidase-B (MAO-B), and endothelial nitric oxide synthase (eNOS) assay kits (Shanghai Shangle Biological Products Research Institute, batch number: 30126078); malondialdehyde (MDA) and superoxide dismutase (SOD) assay kits (Nanjing Jiancheng Biological Products Research Institute, batch number: THZ-C); a constant temperature oscillator (Taicang Experimental Equipment Factory); an Infinite M200 ELIASA (TECAN), 5430R low-temperature high-speed centrifuge (Eppendorf Company, USA), an AL204 electronic balance (Mettler-Toledo Instruments Co. Ltd.), and a Morris water maze apparatus (Chengdu Taimeng Technology Co. Ltd.) were used in this study.

\subsection{Animal Experiments}

2.2.1. Preparation of Cordyceps Polypeptide [18]. An appropriate amount of the dried fruiting body of Cordyceps militaris was ground into powders (40 mesh). The powder was then defatted with petroleum ether and the residue was dried. After drying, $50 \mathrm{~g}$ of the dried residue was dissolved in $450 \mathrm{~mL}$ distilled water and the solution was adjusted to $\mathrm{pH}$ 2.0 with $1 \mathrm{M} \mathrm{HCl}$. Then $10 \mathrm{~g}$ pepsin was added to the solution at $37^{\circ} \mathrm{C}$ for enzymolysis for $5 \mathrm{~h}$. After the enzymolysis, the solution was heated at $100^{\circ} \mathrm{C}$ in a water bath for $10 \mathrm{~min}$ for inactivation of the pepsin. Next it was filtered and subjected to centrifugation for collection of the supernatant and was lyophilized to obtain a Cordyceps polypeptide powder (5.36 g).

2.2.2. Animal Grouping and Administration. One hundred twenty mice were randomly divided into six groups according to treatment: a blank control group, the model group, a positive control (drug) group, a high-dose Cordyceps polypeptide group (900 mg. $\mathrm{kg}^{-1}$ ), a middle-dose Cordyceps polypeptide group (450 $\mathrm{mg} \cdot \mathrm{kg}^{-1}$ ), and a low-dose Cordyceps polypeptide group (225 mg. $\left.\mathrm{kg}^{-1}\right)$. Mice in the positive control group were given the positive control drug Piracetam Tablets at a dose of $600 \mathrm{mg} \cdot \mathrm{kg}^{-1}$ (calculated based on body surface area), and mice in the blank control and model groups received the same volume of distilled water intragastrically once daily for 45 consecutive days.

2.2.3. Animal Model Preparation and Animal Training. Prior to the behavioral testing, the mice were placed in the water maze apparatus and allowed to swim freely for $2 \mathrm{~min}$ on 2 consecutive days, for adaptation of the mice to the environment. One week before the intragastric administration of drug, the water maze test began. At $1 \mathrm{~h}$ after the intragastric administration, the mice in the blank control group were given an intraperitoneal injection of saline $\left(3 \mathrm{~mL} \cdot \mathrm{kg}^{-1}\right)$ and mice in the other groups received an intraperitoneal injection of scopolamine hydrobromide $\left(3 \mathrm{mg} \cdot \mathrm{kg}^{-1}\right)$ for the establishment of an acquired mouse learning and memory impairment model, and $15 \mathrm{~min}$ later the Morris water maze test was carried out for 7 consecutive days.

2.2.4. Morris Water Maze Test. The Morris water maze apparatus consisted of two parts, a cylindrical tube and recording device. The cylindrical tube (diameter $80 \mathrm{~cm}$, height $30 \mathrm{~cm}$ ) was divided into four quadrants (quadrants 1, 2, 3, and 4). A platform with a diameter of $5 \mathrm{~cm}$ was placed at the center of quadrant 3. Before testing, the tube was filled with water up to $11 \mathrm{~cm}$ below the rim. The water temperature was maintained at $20 \pm 2{ }^{\circ} \mathrm{C}$, and an appropriate amount of titanium dioxide was added to the water to make it ivory. The platform was located at $1-2 \mathrm{~cm}$ below the surface of the water. When the test was carried out, the surrounding environment was kept unchanged. 
The Positioning Navigation Test. The test was performed on days 1-6. Before the start of the test, the mice were allowed to stay on the platform for $30 \mathrm{~s}$. Then the mice were placed into the water facing the pool wall in any two quadrants except the quadrant containing the platform. Escape latencies within $120 \mathrm{~s}$ were recorded and average escape latency values were calculated as the evaluation indexes.

On day 7, the spatial search test was carried out. For this text, the platform was removed, and then the mice were placed into the water in a fixed quadrant, facing the wall of the pool. The number of errors made by the mice while crossing the place where the platform had been within $120 \mathrm{~s}$ was recorded, and the average values were calculated as the evaluation indexes.

\subsubsection{Animal Sampling and Biochemical Index Detection} Method. Following the Morris water maze test, the eyeballs of the mice were removed with clean forceps for the collection of $1 \mathrm{~mL}$ of whole blood. The blood samples were centrifuged at $3000 \mathrm{r} / \mathrm{min}$ at $4^{\circ} \mathrm{C}$ for $10 \mathrm{~min}$ to obtain the serum, and the serum samples were stored at $-20^{\circ} \mathrm{C}$ until use. The whole brain tissues of mice were harvested, weighed, and prepared into a $10 \%$ homogenate upon addition of saline at a ratio of $1: 9(\mathrm{w}: \mathrm{v})$ in an ice water bath. The homogenate was centrifuged at $3500 \mathrm{r} / \mathrm{min}$ at $4^{\circ} \mathrm{C}$ for $10 \mathrm{~min}$ to obtain the supernatant, and the supernatant was stored at $-80^{\circ} \mathrm{C}$ until use. In accordance with the instructions for the detection kits, the serum SOD activity and MDA content were measured, the AChE, $\mathrm{Na}^{+}-\mathrm{k}^{+}$-ATP, and eNOS activities were assessed, and the GABA and Glu contents in the brain tissues were determined according to the instructions for the enzymelinked immunosorbent assay kits.

2.3. RNA Extraction and Quality Control. The total liver RNA was extracted with Trizol reagent (Invitrogen, Gaithersburg, MD, USA) by following the operating instructions. The liver tissue was ground into powders under the condition of liquid nitrogen freezing, and the powders were transferred into a $1.5 \mathrm{~mL}$ centrifuge tube in which Trizol (500 mg liver tissue per $0.5 \mathrm{~mL}$ Trizol) had been preadded before the liquid nitrogen completely volatilized. The liver tissue-Trizol homogenate was drawn strongly several times with a $5 \mathrm{~mL}$ disposable syringe until the homogenate was no longer sticky, in order to fully break down the cells and genomic DNA, which was kept at room temperature for use. Chloroform was added to the homogenate in a ratio of $200 \mu \mathrm{L}$ chloroform per $1 \mathrm{~mL}$ Trizol used, which was strongly oscillated for $30 \mathrm{~s}$ to be mixed evenly, kept at room temperature for $5 \mathrm{~min}$, and then centrifuged at $12000 \mathrm{rpm}$ for $15 \mathrm{~min}$ to obtain the supernatant. The supernatant was carefully transferred to another centrifuge tube, and more attention should be paid to drawing the supernatant to avoid taking the mesophase. The supernatant was added with $500 \mathrm{uL}$ isopropanol to be precipitated at room temperature for $10 \mathrm{~min}$; the solution was centrifuged at $12000 \mathrm{rpm}$ for $15 \mathrm{~min}$ and the supernatant was discarded; the residue was added with $1 \mathrm{~mL}$ of $75 \%$ ethanol to wash the RNA, which was oscillated by vortex oscillation, kept at room temperature for $5 \mathrm{~min}$, and then centrifuged at $7500 \mathrm{rpm}$ for $5 \mathrm{~min}$; the supernatant was discarded, and the residue was added with $1 \mathrm{~mL}$ of $75 \%$ ethanol to be washed once again. The residue-ethanol solution was centrifuged at $7500 \mathrm{rpm}$ for $5 \mathrm{~min}$, the supernatant was thrown away, and the residue was dried at room temperature. Please note that the RNA precipitation should not be too dry; otherwise, it will not be dissolved easily.

The RNA was dissolved in an appropriate amount of TE buffer $(10 \mathrm{mmol} / \mathrm{L}$ Tris, pH7.6, $1 \mathrm{mmol} / \mathrm{L}$ EDTA $)$. The total RNA mass was detected by agarose gel electrophoresis and the mRNA was purified in accordance with the instructions of RNeasy Mini Kit (Qiagen, Valencia, CA, USA). According to the kit instructions, the quality of RNA samples was assessed, and the integrity of RNA samples, inhibitors, and DNA pollution were detected. The quality of mRNA was detected by RNA formaldehyde-agarose gel electrophoresis, and the content of RNA was determined by UV spectrophotometry. The same amount of RNA taken from each mouse in each group was mixed and then used for the chip detection.

\subsection{Gene Chip Analysis}

2.4.1. Sample Labeling and Hybridization. Before RNA labeling, the Agilent ND-1000 was used to detect RNA degradation and determine RNA concentration. For gene chip analysis, samples were labeled with the Agilent Quick Amp Labeling kit and hybridized with Agilent SureHyb.

Sample labeling and array hybridization were performed according to the Agilent One-Color Microarray-Based Gene Expression Analysis protocol (Agilent Technology). Briefly, total RNA from each sample was linearly amplified and labeled with Cy3-UTP. The labeled cRNAs were purified by RNeasy Mini Kit (Qiagen). The concentration and specific activity of the labeled cRNAs (pmol Cy3/ $\mu$ g cRNA) were measured by NanoDrop ND-1000. $1 \mu \mathrm{g}$ of each labeled cRNA was fragmented by adding $11 \mu \mathrm{l} 10 \mathrm{x}$ Blocking Agent and $2.2 \mu \mathrm{l}$ of $25 \mathrm{x}$ fragmentation buffer and then heated at $60^{\circ} \mathrm{C}$ for $30 \mathrm{~min}$, and finally $55 \mu \mathrm{L} 2 \mathrm{x}$ GE hybridization buffer was added to dilute the labeled cRNA. $100 \mu \mathrm{L}$ of hybridization solution was dispensed into the gasket slide and assembled to the gene expression microarray slide. The slides were incubated for 17 hours at $65^{\circ} \mathrm{C}$ in an Agilent Hybridization Oven. The hybridized arrays were washed, fixed, and scanned using the Agilent DNA Microarray Scanner (part number G2505C).

2.4.2. Data Acquisition and Standardization. After washing, the chip was scanned with an Agilent DNA Microarray Scanner. The Agilent Feature Extraction software (v11.0.0.1) was used to acquire chip probe signal values, and the Agilent Gene Spring GX v12.1 software was used for standardization of the chip results. Points located outside of the $95 \%$ confidence interval represented differentially expressed genes.

\subsubsection{Significance Analysis of the Functions of Differentially} Expressed Genes. Gene ontology (GO) annotation of the differentially expressed genes was performed using the NCBI Gene Ontology database to identify all the GO categories in which the genes were involved, and Fisher exact test and the $X^{2}$ test were applied to calculate the significance level and 
TABLE 1: Primer pairs for the real-time quantitative PCR.

\begin{tabular}{lccc}
\hline Gene name & Two-way primer sequence & Annealing temperature $\left({ }^{\circ} \mathrm{C}\right)$ & Product size $(\mathrm{bp})$ \\
\hline \multirow{2}{*}{ Pik3r5 } & F: $5^{\prime}$ CGGCTTCTATTACTTCAACTTCCA3' & 60 & 71 \\
& R: $5^{\prime}$ CGGAGGAAGACTTGATAAACAGAC3' & & 60 \\
F: $1 \beta$ & F: 5' CTTCAGGCAGGCAGTATCACTC3' & & 194 \\
Slc18a2 & R: 5' GCAGTTGTCTAATGGGAACGTC3' & & \\
& F: $5^{\prime}$ TCACCAACCCATTCATAGGACT3' & 60 & 168 \\
\hline
\end{tabular}

error rate for each GO. The error rate was then used to calibrate the $P$ values, consequently screening out the significant GO categories reflected by the differentially expressed genes $(P<0.05)[19]$. The experimental results were analyzed using European Bioinformatics Institute (EBI) database [20].

2.4.4. Functional and Biological Pathway Enrichment Analyses of Differentially Expressed Genes Identified by mRNA Expression Chip Using DAVID. From the open DAVID database (https://david.ncifcrf.gov/), 450 genes were submitted within the gene sets for further analysis, and at the same time, the corresponding gene identifier (gene identifier corresponding to the gene name OFFICIAL_GENE_SYMBOL) was selected. The complete genome of the mouse was selected as including the background genes, and then the "Functional Annotation Tool" was applied as the analysis tool, so that the results of the GO enrichment analysis and pathway enrichment analysis of differentially expressed genes could be obtained [21].

2.5. Real-Time Quantitative Polymerase Chain Reaction (PCR) Verification. Real-time quantitative PCR validation of the differentially expression of specific genes associated with the immunomodulation achieved by Cordyceps polypeptide in mice was conducted in strict accordance with the instructions for the Real-time PCR Master Mix (SYBR Green, Toyobo) [22]. The instrument used was the Roche Light Cycler 1.5, and the primer sequences are provided in Table 1.

2.6. Statistical Analysis. Statistical analyses were performed using SPSS 16.0 statistical software (SPSS, Inc.). The single factor analysis of variance and $q$ test of the measurement data were performed with the analysis of variance (ANOVA) program, and least squared differences were used for the pairwise comparison. The ranked data were analyzed by Rid it. $P<0.05$ was considered statistically significant [23].

\section{Experimental Results}

3.1. Effects of Cordyceps Polypeptide on Behavior of Mice with Learning and Memory Impairment. In the positioning navigation test on days 1-6, the escape latency during the first 3 days did not differ among mice in the different groups, but on days 4-6, the escape latency of mice in the model group was significantly prolonged compared with that of mice in the control group $(P<0.01)$, indicating that the model was successfully established. On days 4 and 5, the escape latencies of mice in both the high-dose Cordyceps polypeptide group and the positive drug group were also significantly shortened compared with that of mice in the model group $(P<0.05)$.
By day 6, the escape latencies of mice in all Cordyceps polypeptide-treated groups and the positive drug group were significantly shortened compared with that of mice in the model group $(P<0.05)$. In the spatial search test on day 7 , the number of times that the mice crossed the platform in the model group was significantly less than that in the control group $(P<0.05)$, whereas the numbers of platform crossings in the high-dose Cordyceps polypeptide group and the positive drug group were significantly greater than that in the control group $(P<0.05$; Table 2$)$.

3.2. Effects of Cordyceps Polypeptide on the Serum SOD Activity and MDA Content. Compared with those in the blank control group, the serum SOD activity in the serum of mice was significantly reduced and the MDA content was significantly increased in mice of the model group $(P<$ 0.05 ), further confirming that the model was successfully established. Compared with those in the model group, the serum SOD activity was significantly increased, and the MDA content was significantly reduced in the high-dose Cordyceps polypeptide group and the positive control group $(P<0.05$; Table 3).

3.3. Effects of Cordyceps Polypeptide on AChE, $\mathrm{Na}^{+}-\mathrm{k}^{+}$ATPase, and eNOS Activities and GABA and Glu Contents in the Brain Tissue of Mice. The AChE activity was significantly higher $(P<0.05)$, the $\mathrm{Na}^{+}-\mathrm{k}^{+}$-ATPase and eNOS activities were significantly lower $(P<0.05)$, and the GABA and Glu contents were significantly lower $(P<0.05)$ in the brain tissue of mice in the model group compared with those in the blank control group, also confirming that the model was successfully established. Compared with those in the model group, the AChE activities were significantly decreased $(P<$ $0.05)$, the $\mathrm{Na}^{+}-\mathrm{k}^{+}$-ATPase and eNOS activities were significantly increased, and GABA and Glu contents were significantly increased $(P<0.05)$ in the brain tissue of mice in the high-dose Cordyceps polypeptide group and the positive control group. However, among these indexes, only the $\mathrm{Na}^{+}$$\mathrm{k}^{+}$-ATPase activity was significantly increased in the brain tissue of mice in low-dose Cordyceps polypeptide group compared with that in the model group $(P<0.05)$. The corresponding data are presented in Table 4.

3.4. $m R N A$ Chip Results. As shown in Figure 1, compared with the model group, there were 450 differentially expressed genes in the brain tissue of mice in the Cordyceps polypeptidetreated group, of which the expression of 175 genes $(38.89 \%)$ was significantly upregulated and that of 275 gene $(61.11 \%)$ 
TABLE 2: Morris water maze test results.

\begin{tabular}{|c|c|c|c|c|}
\hline \multirow{2}{*}{ Group } & \multicolumn{3}{|c|}{ Escape latency (s) } & \multirow{2}{*}{$\begin{array}{c}\text { Crossing the platform (times) } \\
\text { 7th day }\end{array}$} \\
\hline & 4th day & 5 th day & 6th day & \\
\hline $\mathrm{CON}$ & $38.12 \pm 34.65$ & $33.26 \pm 22.16$ & $30.17 \pm 22.80$ & $4.20 \pm 3.65$ \\
\hline M & $85.56 \pm 43.75^{* *}$ & $74.33 \pm 50.61^{* *}$ & $71.14 \pm 50.13^{* *}$ & $1.54 \pm 1.63^{*}$ \\
\hline $\mathrm{L}-\mathrm{CP}$ & $74.55 \pm 49.26$ & $52.62 \pm 49.60$ & $51.07 \pm 44.34^{\#}$ & $2.54 \pm 2.47$ \\
\hline $\mathrm{M}-\mathrm{CP}$ & $78.87 \pm 48.13$ & $54.87 \pm 43.41$ & $49.98 \pm 35.91^{\#}$ & $1.93 \pm 2.02$ \\
\hline $\mathrm{H}-\mathrm{CP}$ & $59.86 \pm 51.48^{\#}$ & $47.30 \pm 47.55^{\#}$ & $39.61 \pm 39.12^{\#}$ & $3.25 \pm 1.86^{\#}$ \\
\hline PC & $58.23 \pm 40.16^{\#}$ & $48.64 \pm 39.73^{\#}$ & $38.14 \pm 26.66^{\#}$ & $2.64 \pm 3.22^{\#}$ \\
\hline
\end{tabular}

${ }^{*} P<0.05$ versus blank control group, ${ }^{* *} P<0.05$ versus blank control group, ${ }^{\#} P<0.05$ versus model group; CON: blank control group; M: model group; L-CP: low-dose CP group; M-PC: middle-dose CP group; H-CP: high-dose CP group; PC: positive control group.

TABLE 3: Effects of Cordyceps polypeptide on the serum SOD activity and MDA content in mice.

\begin{tabular}{lcc}
\hline Group & SOD $(\mathrm{U} / \mathrm{mL})$ & $\mathrm{MDA}(\mathrm{nmol} / \mathrm{mL})$ \\
\hline CON & $128.72 \pm 17.13$ & $5.15 \pm 0.91$ \\
M & $105.19 \pm 12.38^{*}$ & $6.48 \pm 0.86^{*}$ \\
L-CP & $116.73 \pm 13.16$ & $5.57 \pm 1.08$ \\
M-CP & $118.20 \pm 25.22$ & $5.66 \pm 0.89$ \\
H-CP & $127.57 \pm 18.35^{\#}$ & $4.99 \pm 1.51^{\#}$ \\
PC & $132.44 \pm 16.48^{\#}$ & $5.16 \pm 0.94^{\#}$ \\
\hline
\end{tabular}

${ }^{*} P<0.05$ versus blank control group, ${ }^{\#} P<0.05$ versus model group; CON: blank control group; M: model group; L-CP: low-dose CP group; M-PC: middledose CP group; H-CP: high-dose CP group; PC: positive control group.

TABLE 4: Effects of Cordyceps polypeptide on AChE, $\mathrm{Na}^{+}-\mathrm{k}^{+}$-ATPase, and eNOS activities and GABA and Glu contents in brain tissue of mice.

\begin{tabular}{|c|c|c|c|c|c|}
\hline Group & $\operatorname{AchE}(\mathrm{ng} / \mathrm{mL})$ & $\mathrm{Na}^{+}-\mathrm{k}^{+}-\mathrm{ATP}(\mathrm{ng} / \mathrm{mL})$ & eNOS (U/mL) & GABA (ng/mL) & Glu (nmol/mL) \\
\hline $\mathrm{CON}$ & $15.41 \pm 0.71$ & $16.42 \pm 1.65$ & $4.62 \pm 0.50$ & $85.73 \pm 5.87$ & $60.05 \pm 3.71$ \\
\hline M & $16.63 \pm 0.87^{*}$ & $13.87 \pm 2.11^{*}$ & $4.04 \pm 0.45^{*}$ & $76.33 \pm 8.80^{*}$ & $4.77 \pm 5.14^{*}$ \\
\hline $\mathrm{L}-\mathrm{CP}$ & $15.64 \pm 0.94$ & $16.96 \pm 2.18^{\#}$ & $4.60 \pm 0.55$ & $84.61 \pm 4.46^{\#}$ & $58.25 \pm 3.63$ \\
\hline $\mathrm{M}-\mathrm{CP}$ & $15.59 \pm 0.75$ & $16.11 \pm 2.83$ & $4.62 \pm 0.63$ & $84.60 \pm 7.99$ & $58.13 \pm 4.93$ \\
\hline $\mathrm{H}-\mathrm{CP}$ & $15.43 \pm 0.90^{\#}$ & $16.77 \pm 1.81^{\#}$ & $4.65 \pm 0.44^{\#}$ & $87.98 \pm 6.45^{\#}$ & $60.71 \pm 1.09^{\#}$ \\
\hline PC & $15.35 \pm 0.56^{\#}$ & $17.34 \pm 3.17^{\#}$ & $4.70 \pm 0.66^{\#}$ & $85.24 \pm 4.99^{\#}$ & $60.98 \pm 4.34^{\#}$ \\
\hline
\end{tabular}

${ }^{*} P<0.05$ versus blank control group, ${ }^{\#} P<0.05$ versus model group; CON: blank control group; M: model group; L-CP: low-dose CP group; M-PC: middledose $\mathrm{CP}$ group; $\mathrm{H}-\mathrm{CP}$ : high-dose $\mathrm{CP}$ group; PC: positive control group.

was significantly downregulated. The differences in the mRNA chip results were significant between the Cordyceps polypeptide-treated group and the model group, and the results showed that Cordyceps polypeptide could significantly inhibit the expression level of genes related to the nervous system (the expression of $61.11 \%$ genes was downregulated).

3.5. Clustering Results. The GO cluster analysis of the 450 differentially expressed genes was carried out using the DAVID database, and 36 related pathways were found. Seven of these pathways were directly related to the immune function. Statistical analysis for the genes related to these seven pathways showed that multiple genes were involved in more than one of the seven pathways, and further analysis revealed that the pathways involving three genes (PIK3R5, IL$1 \mathrm{~B}$, and SLC18A2) were the most (Table 5).

3.6. Real-Time Quantitative PCR Verification. Real-time quantitative PCR validation of the expression of three genes, including Pik3r5, Il-1 $\beta$, and Slc18a2, was conducted. As shown in Table 6, the Pik3r5 and $I l-1 \beta$ expression levels were found to be significantly altered in the brain tissue of mice in the model group $(P=0.015997, P=0.001177)$ with expression ratios of 0.8 and 0.12 , respectively, between the Cordyceps polypeptidetreated group and model group (Figure 2), and this resulted in negative regulation on immune activity. The Slc18a2 gene was significantly upregulated in the brain tissue of mice with learning and memory impairment $(P=0.002095$ with an expression ratio of 1.79 between the Cordyceps polypeptidetreated group and model group).

\section{Discussions}

With increases in the social pressure and the incidence of various diseases, more and more people, including not only the elderly but also teens, have begun to experience the symptoms of a decline in memory. Therefore, there is a need for drugs or active components that can improve learning and memory. Studies have shown that Cordyceps militaris enhances immunity combat aging and promotes 
TABLE 5: Screened out representative genes.

\begin{tabular}{|c|c|c|c|}
\hline No. & Genes name & Pathway count & Pathway name \\
\hline 1 & Pik3r5 & 4 & $\begin{array}{c}\text { Chagas disease (American trypanosomiasis) natural } \\
\text { toxoplasmosis } \\
\text { Leukocyte transendothelial migration } \\
\text { Dopaminergic synapse }\end{array}$ \\
\hline 2 & $I l-1 \beta$ & 3 & $\begin{array}{c}\text { Chagas disease (American trypanosomiasis) natural } \\
\text { toxoplasmosis } \\
\text { Prion diseases }\end{array}$ \\
\hline 3 & Slc18a2 & 3 & $\begin{array}{c}\text { Chagas disease (American trypanosomiasis) } \\
\text { Leukocyte transendothelial migration } \\
\text { Dopaminergic synapse }\end{array}$ \\
\hline
\end{tabular}
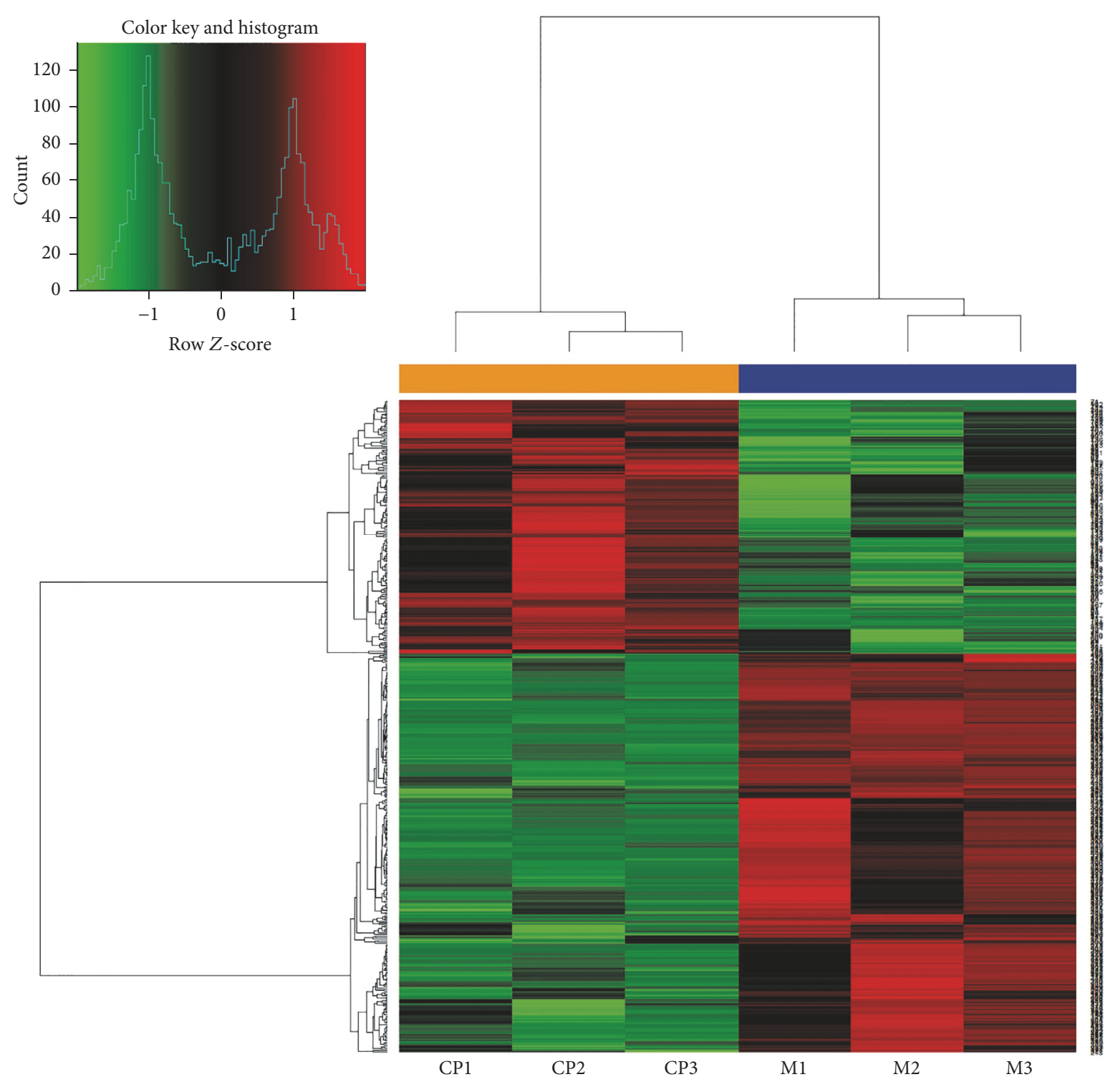

$\square 0$

FIGURE 1: Results of mRNA expression profile microarray analysis (M: model group; CP: Cordyceps polypeptide group). 

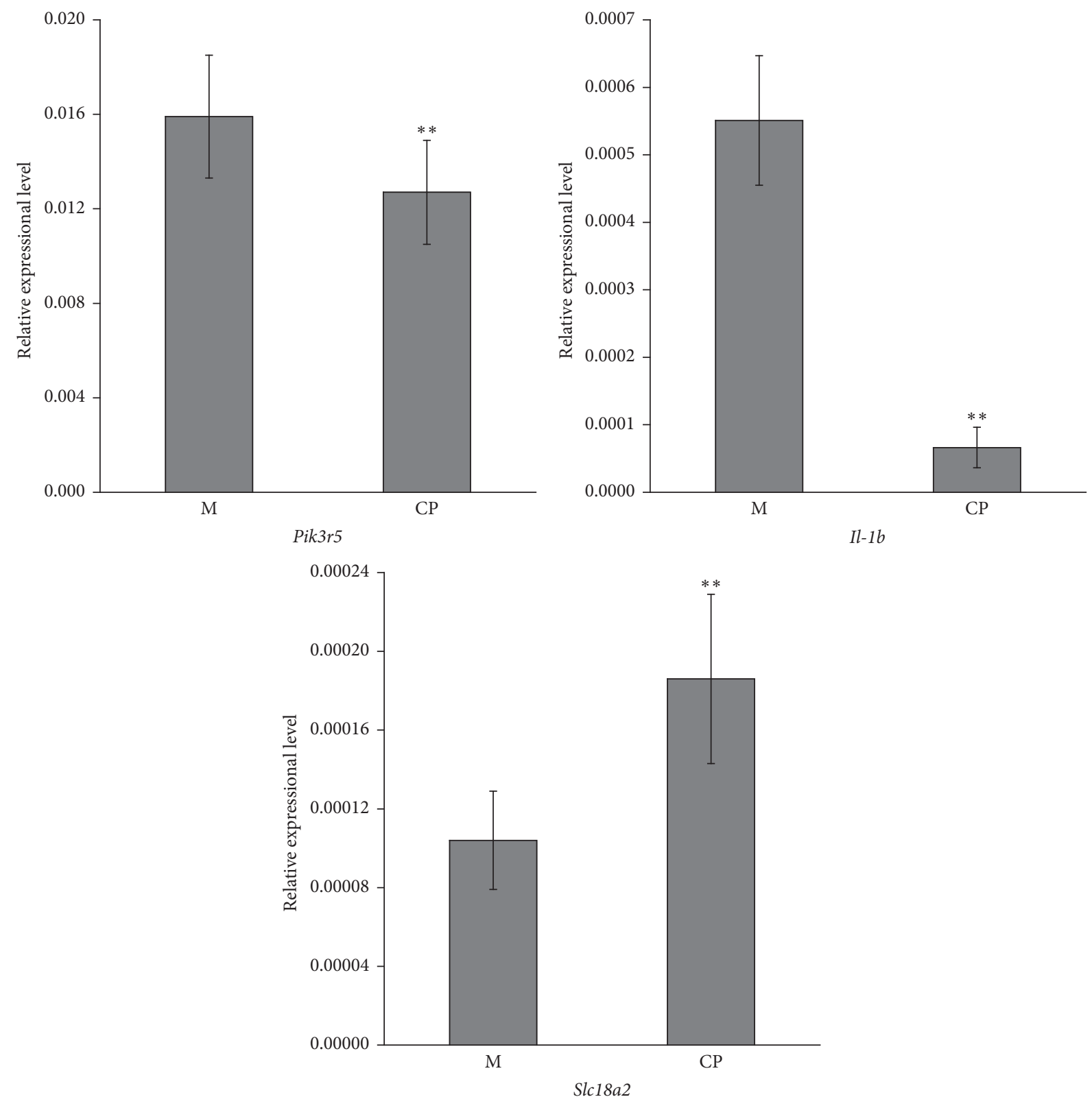

FIGURE 2: Real-time quantitative PCR validation of Pik3r5, Il-1b, and Slc18a2 gene (M: model group; CP: Cordyceps polypeptide group; ${ }^{* *} \mathrm{P}<$ $0.01)$.

TABLE 6: Experimental results of real-time quantitative PCR.

\begin{tabular}{lccc}
\hline Data comparison scheme & Pik3r5 & $I l-1 b$ & Slc18a2 \\
\hline $\mathrm{M}$ & $1.59 E-02$ & $5.51 E-04$ & $1.04 E-04$ \\
$\mathrm{CP}$ & $1.27 E-02$ & $6.62 E-05$ & $1.86 E-04$ \\
$\mathrm{CP} / \mathrm{M}$ & 0.80 & 0.12 & 1.79 \\
$P$ value & 0.0002353 & 0.001177 & 0.002095
\end{tabular}

M: model group; CP: Cordyceps polypeptide group.

cardiovascular regulation, but there have been few reports on the effects of Cordyceps polypeptide on learning and memory to our knowledge. In the present study, scopolamine hydrobromide was used to establish a mouse model of learning and memory impairment, and the effect of Cordyceps polypeptide on the learning and memory in these mice was investigated from two perspectives, that is, the behavioral one in the Morris water maze test and the molecular detection of related biochemical indexes. The Morris water maze testing showed that Cordyceps polypeptide could shorten the latency for finding the platform and increase the number of crossings at the place where the platform was previously placed, indicating that Cordyceps polypeptide could improve the learning and memory ability of mice with learning and memory impairment induced by scopolamine.

SOD is involved in the scavenging of oxygen free radicals in the body and thereby prevents lipid peroxidation and protects the cell membrane from oxidative damaged [24]. 
MDA is one of the products of lipid peroxidation induced upon injury and can aggravate the damage to the cell membrane. Therefore, measurement of the SOD activity and MDA content can be used to evaluate the antioxidant effects. $\mathrm{AChE}$ is a neurotransmitter that is closely associated with the function of learning and memory, and an increase in AChE content can damage the cholinergic nerve, causing a cognitive impairment, which means AChE can be used as an indicator for revaluating the learning and memory [25]. GABA and Glu are the central inhibitory and stimulatory neurotransmitters, respectively, and a balance in their expression plays an important role in the maintenance of normal functions of the central nervous system. $\mathrm{Na}^{+}-\mathrm{k}^{+}$-ATPase, also known as sodium potassium pump, is located in the cell membrane and involved in the energy supply. Moreover it can increase cerebral blood flow, promote brain energy metabolism, and improve learning and memory functions. eNOS catalyzes the in vivo synthesis of NO with a protective effect on the nervous system [26]. Promoting the activity of eNOS can increase the release of NO to protect nerve cells. Our results showed that Cordyceps polypeptide could improve the serum SOD activity, decrease the MDA content and AChE activity in the mouse brain, increase $\mathrm{Na}^{+}-\mathrm{k}^{+}$-ATPase and eNOS activity, and increase GABA and Glu expression, suggesting that Cordyceps polypeptide could improve the capacity for learning and memory in mice with a learning and memory disorder.

In the present study, through mRNA expression microarray analysis, a total of 450 differentially expressed genes were identified between the Cordyceps polypeptide-treated group and the model group. Then 36 related pathways were revealed through the clustering analysis for gene function, and the pathways involving three genes Pik3r5, Il-1 $\beta$, and Slc18a2 existed were the most. The real-time quantitative PCR analysis of the expression of Pik3r5, Il-1 $\beta$, and Slc18a2 further indicated that these three genes were all the key factors in the effects of Cordyceps polypeptide on the nervous system in the mouse model of learning and memory impairment.

Il-1 $\beta$ is a cytokine secreted by $\mathrm{T}$ helper 1 (Th1) cells to promote inflammation. Studies have shown that $I l-1 \beta$ may be involved in the pathophysiological process of depression, and the response to antidepressant treatment involves reducing the function of the 5-HT system, activating the hypothalamic-pituitary-adrenal (HPA) axis, and affecting the regeneration of neurons [27]. Additional reports have demonstrated that childhood trauma may increase the level of cytokines in vivo and influence the course of depression and the therapeutic effect of antidepressive drugs. Our mRNA chip analysis in this study confirms that Cordyceps polypeptide can inhibit the expression of a gene, which may be advantageous for the regeneration of neurons and play a protective role in the nervous system, whereby Cordyceps polypeptide may improve the mice's learning and memory of mice [28].

The Slc18a2 gene is located at chromosomal region 10q25.3 and encodes vesicular monoamine transporter 2 (VMAT2) which can transport dopamine. It also encodes epinephrine and serotonin which cross the membrane into synaptic vesicles and take part in the monoamine metabolism. It has been reported that the physiological roles of these monoamines are associated with motor control, mood stability, and autonomic functions. Normal functioning of this process is essential to maintain dopamine metabolism in substantia nigra neurons.

Slc18a2 gene expression was reported to reduce the dyskinesia and depression-like behavior in mouse models [29]. Slc18a2 gene expression was also shown to promote the secretion of neurotransmitters when the gene chip technology was applied to observe the effect of arsenic on the expression profile of genes related to the neurotransmitters in the cerebellum of mice. In the present study, Cordyceps polypeptide treatment could promote the expression of Slc18a2 and thereby promoted the secretion of neurotransmitters in mice to reduce dyskinesia and depression-like behavior, which may be conducive to the improvement of learning and memory.

The phosphatidylinositol-3-kinase (PI3K)/Akt and mammalian target of rapamycin (mTOR) pathway, one of the most important intracellular signal transduction pathways, plays important roles in the processes of cell growth, survival, proliferation, and apoptosis as well as in angiogenesis [30]. PI3K family members are important kinases of inositol and phosphatidylinositol as well as important signal transduction molecules, involved in the regulation of cell proliferation, apoptosis, and differentiation process [31]. PIK3R5, a member of the PI3K family, has been rarely studied, and there has been no study linking it to the nervous system [32]. In the present study, real-time quantitative PCR demonstrated significant downregulation of Pik3r5 $(P=0.0002353)$ with Cordyceps polypeptide treatment, and the experimental results of it were more significant than those of the other 2 genes. The downregulation expression in mRNA chip indicates that Cordyceps polypeptide may inhibit the expression of the gene, which can be used as a potential drug target of Cordyceps militaris for the further experiment research.

\section{Conclusions}

A mouse model of learning and memory impairment was established via injection of scopolamine hydrobromide and the effects of different doses of Cordyceps polypeptide delivered intragastrically were investigated by the evaluation of mouse behavior via Morris water maze testing, the measurement of several biological indexes in the serum and brain tissue of the mice, and the identification of differentially expressed genes and the related cellular signaling pathways. The results showed that Cordyceps polypeptide could improve the learning and memory in these mice, increase the serum SOD activity, decrease the serum MDA content, reduce AChE activity, increase $\mathrm{Na}^{+}-\mathrm{k}^{+}$-ATPase and eNOS activity, and increase GABA and Glu levels in the brain tissue of the mice. The mRNA expression file chip analysis, clustering analysis of the gene function according to the DAVID database, and real-time qPCR verification indicate that the improvement in learning and memory induced by Cordyceps polypeptide treatment in the mouse model of learning and memory impairments is likely related to its effects on Pik3r5, Il-1 1 , and Slc18a2, and these three genes may be the potential targets for Cordyceps militaris. 


\section{Conflicts of Interest}

The authors declare that they have no conflicts of interest.

\section{Acknowledgments}

This work was financially supported by National and Local United Engineering R\&D Center of Research and Development on Active Peptide of Medicinal Plants and Animals in Chang Bai Mountains, the Jilin Province Science and Technology Development project (201603095YY, 201603092YY, 20170307016YY), Jilin City Science and Technology Innovation and Development project (20166017), Jilin Province Chinese Medicine Science and Technology projects (2017086), the research project of Jilin Provincial Education Department (JJKH20170064KJ, JJKH20170065KJ), Beihua University Research and Development Innovation Team of Animal and Plant Resources in Changbai Mountain, and Beihua University Young Teacher Promotion Program (Beihua University (2016) 43).

\section{References}

[1] Kempermann G. and Gage F. H., "Genetic determinants of adult hippcampalneu-rogenesis correlate with acquisition,but not ProbetrialPerformance,in the wat erMazetask," European Journal of Neuroscience, vol. 16, no. 1, pp. 129-136, 2002.

[2] H. van Praag, T. Shubert, C. Zhao, and F. H. Gage, "Exercise enhances learning and hippocampal neurogenesis in aged mice," The Journal of Neuroscience, vol. 25, no. 38, pp. 8680-8685, 2005.

[3] J. S. Snyder, N. S. Hong, R. J. McDonald, and J. M. Wojtowicz, "A role for adult neurogenesis in spatial long-term memory," Neuroscience, vol. 130, no. 4, pp. 843-852, 2005.

[4] L. A. Meltzer, R. Yabaluri, and K. Deisseroth, "A role for circuit homeostasis in adult neurogenesis," Trends in Neurosciences, vol. 28, no. 12, pp. 653-660, 2005.

[5] G. Kempermann, L. Wiskott, and F. H. Gage, "Functional significance of adult neurogenesis," Current Opinion in Neurobiology, vol. 14, no. 2, pp. 186-191, 2004.

[6] J. Song, Y. Wang, C. Liu et al., "Cordycepsmilitaris fruit body extract ameliorates membranous glomerulonephritis by attenuating oxidative stress and renal inflammation via the NFkappaB pathway," Food \& Function, vol. 7, no. 4, pp. 2006-2015, 2006.

[7] G. F. Zhou, Q. T. Liu, B. Zhou et al., “The potential molecular effects of bursal septpeptide II on immune induction and antitumor activity," Journal of Veterinary Science, vol. 16, no. 3, pp. 325-331, 2015.

[8] A. Kasus-Jacob, S. Noor-Mohammadi, G. L. Griffith, H. Hinsley, L. Mathias, and H. A. Pereira, "A multifunctional peptide based on the neutrophil immune defense molecule, CAP37, has antibacterial and wound-healing properties," Journal of Leukocyte Biology, vol. 97, no. 2, pp. 341-350, 2015.

[9] K. Izgi, B. Iskender, C. Sakalar, A. Arslanhan, B. Saraymen, and H. Canatan, "Evaluation of two different adjuvants with immunogenic uroplakin 3A-derived peptide for their ability to evoke an immune response in mice," European Cytokine Network, vol. 26, no. 2, pp. 46-56, 2015.

[10] J.-Y. Liu, C.-P. Feng, X. Li, M.-C. Chang, J.-L. Meng, and L.-J. Xu, "Immunomodulatory and antioxidative activity of Cordyceps militaris polysaccharides in mice," International Journal of Biological Macromolecules, vol. 86, pp. 594-598, 2016.
[11] J. Song, Y. Wang, and M. Teng, "Cordyceps militaris induces tumor cell death via the caspasedependent mitochondrial pathway in HepG2 and MCF7 cells," Molecular Medicine Reports, vol. 13, no. 6, pp. 5132-5140, 2016.

[12] A. V. Avtonomova, L. M. Krasnopolskaya, M. I. Shuktueva, E. B. Isakova, and V. M. Bukhman, "Assessment of Antitumor Effect of Submerged Culture of Ophiocordycepssinensis and Cordycepsmilitaris," AntibiotKhimioter, vol. 60, no. 7-8, pp. 1417, 2016.

[13] C. P. Chiu, S. C. Liu, C. H. Tang et al., "Anti-inflammatory Cerebrosides from Cultivated Cordycepsmilitaris," Journal of Agricultural and Food Chemistry, vol. 64, no. 7, pp. 1540-1548, 2016.

[14] W. S. Jo, Y. J. Choi, H. J. Kim et al., “The Anti-inflammatory Effects of Water Extract from," Mycobiology, vol. 38, no. 1, pp. 46-51, 2010.

[15] L. Xie, L. An, P. Du et al., "Study on the enzymolysis technology of Cordyceps militaris peptides and its effect on immune function in mice," Chinese Traditional Patent Medicine, vol. 38, no. 9, pp. 2048-2050, 2016.

[16] L. F. Dos Santos, R. Rubel, S. J. Ribeiro Bonatto et al., "Effects of Cordyceps sinensis on macrophage function in high-fat diet fed rats and its anti-proliferative effects on IMR-32 human neuroblastoma cells," Journal of Ethnopharmacology, vol. 214, pp. 106-112, 2018.

[17] D. Wang, J. Wang, D. Wang et al., "Neuroprotective Effects of Butanol Fraction of Cordyceps cicadae on Glutamate-Induced Damage in PC12 Cells Involving Oxidative Toxicity," Journal of Chromatography. B, Analytical Technologies in the Biomedical and Life Sciences, vol. 1, pp. 1061-1062, 2017.

[18] L. Xie, L. An, and P. Du, "Study on the preparation method of enzymatic hydrolysis process of Cordyceps militaris active peptides," Lishizhen Medicine and Materia Medica Research, vol. 28, no. 5, pp. 1123-1125, 2017.

[19] J. Ye, L. Fang, H. Zheng et al., "WEGO: a web tool for plotting GO annotations," Nucleic Acids Research, vol. 34, pp. W293W297, 2006.

[20] P. Rodriguez-Tomé, P. J. Stoehr, G. N. Cameron, and T. P. Flores, "The European Bioinformatics Institute (EBI) databases," Nucleic Acids Research, vol. 24, no. 1, pp. 6-12, 1996.

[21] D. W. Huang, B. T. Sherman, Q. Tan et al., "The DAVID gene functional classification tool: a novel biological module-centric algorithm to functionally analyze large gene lists," Genome Biology, vol. 8, no. 9, article R183, 2007.

[22] M. E. De Almeida, O. Koru, F. Steurer, B. L. Herwaldt, and A. J. Da Silva, "Detection and differentiation of Leishmania spp. In clinical specimens by use of a SYBR green-based real-time PCR assay," Journal of Clinical Microbiology, vol. 55, no. 1, pp. 281-290, 2017.

[23] Q. Shi, W. Zhou, C. Chen et al., "The features of genetic prion diseases based on Chinese surveillance program," PLOS ONE, vol. 10, no. 10, Article ID e0139552, 2015.

[24] M. Zamanian, M. R. Hajizadeh, A. Esmaeili Nadimi, A. Shamsizadeh, and M. Allahtavakoli, "Antifatigue effects of troxerutin on exercise endurance capacity, oxidative stress and matrix metalloproteinase-9 levels in trained male rats," Fundamental \& Clinical Pharmacology, vol. 31, no. 4, pp. 447-455, 2017.

[25] J. Deguil, L. Ravasi, Y. Lamberty et al., "Early development of symptomatic drugs in $\mathrm{AD}$ : A systematic review of the use of biomarkers," CNS and Neurological Disorders - Drug Targets, vol. 15, no. 7, pp. 823-836, 2016.

[26] C. Bocca, E. Novo, A. Miglietta, and M. Parola, "Angiogenesis and Fibrogenesis in Chronic Liver Diseases," Cellular and 
Molecular Gastroenterology and Hepatology, vol. 1, no. 5, pp. 477-488, 2015.

[27] M. Gu, K. Miyoshi, R. Dubner et al., "Spinal 5-HT3 receptor activation induces behavioral hypersensitivity via a neuronalglial-neuronal signaling cascade," The Journal of Neuroscience, vol. 31, no. 36, pp. 12823-12836, 2011.

[28] F. Xue, L. Liu, J. Fan et al., "Interleukin-1 $\beta$ promotes the neurogenesis of carotid bodies by stimulating the activation of ERK1/ 2," Respiratory Physiology \& Neurobiology, vol. 219, pp. 78-84, 2015.

[29] S. P. Alter, K. A. Stout, K. M. Lohr et al., "Reduced vesicular monoamine transport disrupts serotonin signaling but does not cause serotonergic degeneration," Experimental Neurology, vol. 275, pp. 17-24, 2016.

[30] L. C. Cantley, “The phosphoinositide 3-kinase pathway," Science, vol. 296, no. 5573, pp. 1655-1657, 2002.

[31] K. M. Nicholson and N. G. Anderson, "The protein kinase B/ Akt signalling pathway in human malignancy," Cellular Signalling, vol. 14, no. 5, pp. 381-395, 2002.

[32] C. Thauvin-Robinet, M. Auclair, L. Duplomb et al., "PIK3R1 mutations cause syndromic insulin resistance with lipoatrophy," American Journal of Human Genetics, vol. 93, no. 1, pp. 141-149, 2013. 


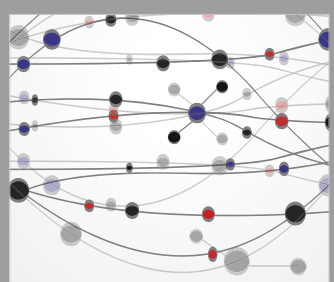

The Scientific World Journal


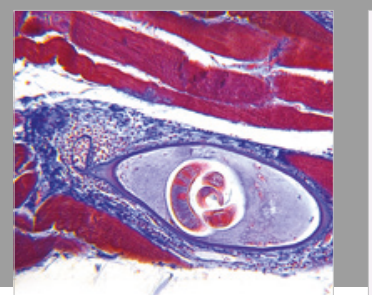

Gastroenterology Research and Practice

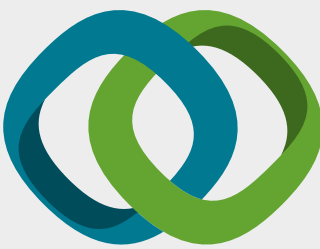

\section{Hindawi}

Submit your manuscripts at

www.hindawi.com
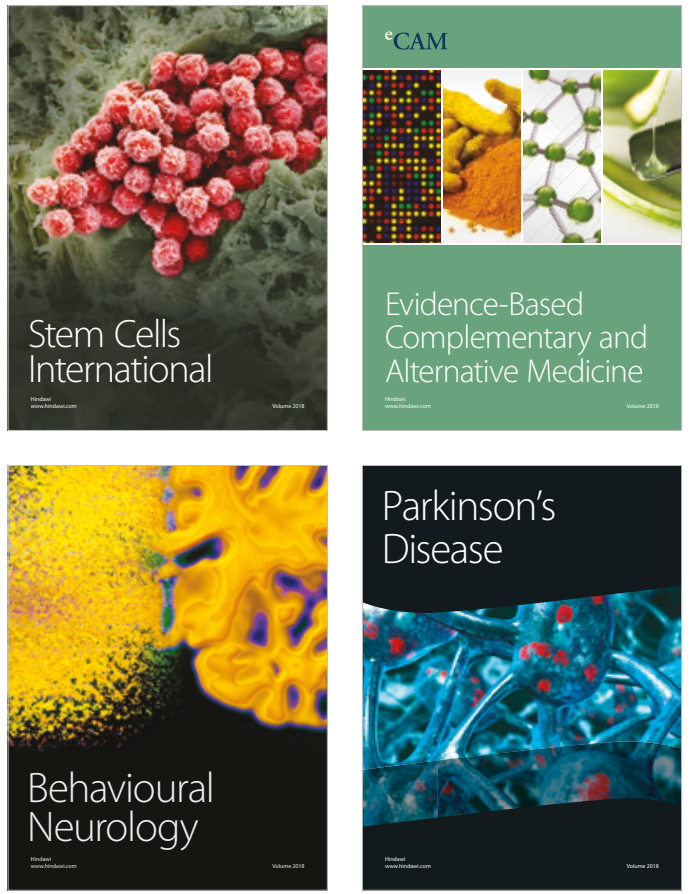

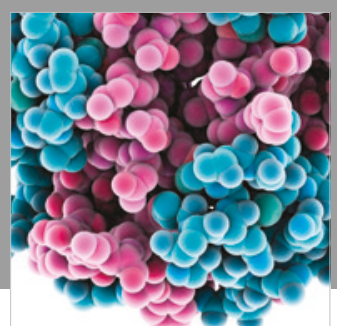

ournal of

Diabetes Research



Disease Markers
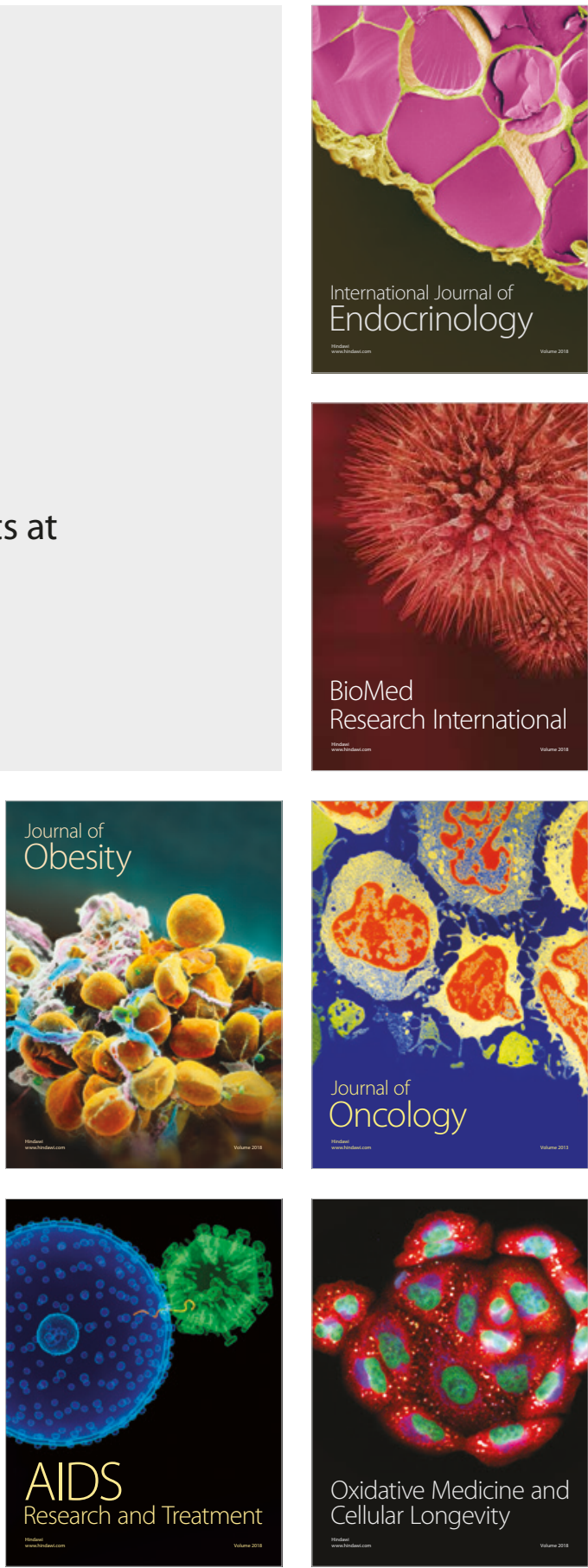